\title{
VISCOELÁSTICOS OFTÁLMICOS: COMPARAÇÃO ENTRE OS COMERCIAIS E FORMULAÇÕES DE GALACTOMANANA DE Dimorphandra gardneriana
}

\author{
Natália R. Pires, Pablyana L. R. da Cunha, Regina C. M. de Paula e Judith P. A. Feitosa* \\ Departamento de Química Orgânica e Inorgânica, Universidade Federal do Ceará, CP 6021, 60455-760, Fortaleza - CE, Brasil \\ Francisco V. F. Jamacaru e Manoel O. Moraes Filho
}

Depto de Fisiologia e Farmacologia, Universidade Federal do Ceará, 60430-270 Fortaleza - CE, Brasil

Recebido em 11/12/09; aceito em 4/5/10; publicado na web em 21/9/10

\begin{abstract}
OPHTALMIC VISCOSURGICAL DEVICES: COMPARISON BETWEEN COMMERCIAL AND FORMULATIONS BASED ON Dimorphandra gardneriana GALACTOMANNAN. Ophthalmic viscosurgical devices (OVD) are materials injected in intraocular space during cataract removal to reduce trauma in the patient's eye. Three Brazilian commercially available OVDs (Medilon, Metilcelulose ${ }^{\circledR}$ and $\mathrm{Ofthyal}^{\circledR}$ ) were evaluated as well as formulations based on Dimorphandra gardneriana galactomannan. Viscosity and viscoelastic parameters, such as viscosity at zero shear, pseudoplasticity index, elastic and viscous moduli, relaxation time, were determined and compared. Characteristics of an effective OVD were proposed. None of the Brazilian devices studied fulfill the rheological requirements. Only the galactomannan at $3 \%$ concentration showed potential to be used as effective OVD.
\end{abstract}

Keywords: fava danta rheology; ophthalmic viscosurgical device; cataract.

\section{INTRODUÇÃO}

A catarata é um distúrbio ocular caracterizado pela opacificação parcial ou total do cristalino. Esse distúrbio é a principal causa mundial de cegueira, sendo responsável por $48 \%$ dos casos de incapacidade visual. ${ }^{1}$ No Brasil, a situação é semelhante, sendo a catarata responsável por $40-50 \%$ das causas de cegueira em adultos. ${ }^{2}$ Com o aumento da expectativa de vida e o consequente incremento na população de idosos, prevê-se um aumento de casos. ${ }^{3}$

A extração do cristalino cataratoso e o implante de uma lente intraocular artificial são altamente eficientes para corrigir o distúrbio visual. A facoemulsificação é a técnica cirúrgica atualmente utilizada pela maioria dos cirurgiões para a remoção da catarata. ${ }^{4}$ Ela consiste na fragmentação ultrassônica e emulsificação do núcleo do cristalino opacificado, preservando-se a sua cápsula, mediante uma incisão corneana mínima. Para facilitar o procedimento, substâncias viscoelásticas, conhecidas como dispositivos viscocirúrgicos oftálmicos (ophthalmic viscosurgical device, OVD), são injetadas na câmara anterior do olho. Após a facoemulsificação do núcleo, realiza-se a aspiração do material cortical. Em seguida, implanta-se uma lente intraocular na bolsa formada pela cápsula remanescente do cristalino. Por fim, efetua-se a remoção do fluido viscoelástico injetado. ${ }^{5,6} \mathrm{As}$ sim, as principais finalidades dos OVDs são auxiliar na manutenção da câmara anterior durante o procedimento cirúrgico, facilitando as manobras operatórias, e proteger os tecidos oculares, sobretudo as células endoteliais da córnea, dos danos causados pelo processo de facoemulsificação. ${ }^{6}$

Importantes características dos OVDs, adequadas às diferentes etapas do processo cirúrgico são de natureza reológica. Os parâmetros mais significativos são: viscosidade a cisalhamento zero, $\eta_{0}$, pseudoplasticidade, propriedades viscoelásticas e tempo de relaxação. ${ }^{7} \mathrm{~A}$ habilidade do OVD de manter o espaço quando o tecido ocular estiver em repouso é refletida pelo $\eta_{0}$. A resistência oferecida pelo OVD ao movimento normal dos instrumentos cirúrgicos no olho depende da viscosidade em taxas de cisalhamento intermediárias (1-10 $\left.\mathrm{s}^{-1}\right)$. A resistência do OVD em fluir através da pequena cânula utilizada na

*e-mail: judith@dqoi.ufc.br aspiração é indicada pela viscosidade em altas taxas de cisalhamento (da ordem de $10^{3} \mathrm{~s}^{-1}$ ).

O OVD ideal deve apresentar, então, alta viscosidade em baixas taxas de cisalhamento ou mesmo em repouso (propriedades ditas coesivas), com o propósito de auxiliar na manutenção da câmara anterior. Ademais, deve possuir baixa viscosidade em altas taxas para diminuir a resistência ao fluxo durante as manobras cirúrgicas, como a inserção e remoção de instrumentos ou a aspiração de líquidos, assim como para propiciar uma cobertura mais uniforme do endotélio corneano, conferindo-lhe, por conseguinte, maior proteção (propriedades dispersivas). $., 8,9$

Atualmente, vários OVDs estão disponíveis no mercado internacional. Eles incluem preparações de polímeros naturais, como os polissacarídeos (hialuronato de sódio, NaHA; sulfato de condroitina, SC) e seus derivados (hidroxipropilmetilcelulose, HMPC), ${ }^{9} \mathrm{em}$ concentrações que variam entre 1 e $3 \% .{ }^{10} \mathrm{~A}$ linha Healon, produtos à base de NaHA, custa US $\$ 191,19$, por uma seringa de $0,85 \mathrm{~mL},{ }^{11} \mathrm{o}$ OcuCoat $^{\circledR}$, à base de HMPC, custa US $\$ 520,45$ por caixa com 6 seringas de $1 \mathrm{~mL},{ }^{12}$ e o Viscoat ${ }^{\oplus}$, à base de NaHA e SC, custa US\$151,99 por cada $0,5 \mathrm{~mL},{ }^{13}$ no mercado internacional. Isso torna inviável a utilização desses OVDs no sistema público e, também, para os clientes de operadoras de planos de saúde. ${ }^{14}$ Outras opções de mais baixo custo são produzidas e utilizadas no Brasil. As mais comuns são as de hidroxipropilcelulose 2\% (Metilcelulose ${ }^{\circledR}$ da Ophthalmos) ${ }^{15,16} \mathrm{e}$ 1,5\% poliacrilamida (Medilon ${ }^{\circledR}$ da Mediphacos Ophthalmic Professionals). O primeiro custa $\mathrm{R} \$ 42,50$ a seringa de $1,5 \mathrm{~mL}^{17}$ e o segundo $\mathrm{R} \$ 60,00$ a seringa de $1 \mathrm{~mL} .{ }^{18}$ Outro OVD nacional é à base de ácido hialurônico (Ofthyal ${ }^{\circledR}$ ), cujos estudos reológicos, assim como os dois outros OVDs nacionais citados, não estão disponíveis na literatura.

As galactomananas são polissacarídeos neutros, encontrados em sementes da família Leguminosae. São solúveis em água e formam soluções viscosas mesmo em baixas concentrações. Possuem uma cadeia principal de manose unida por ligações $\beta-1,4$ com ramificações de galactose com ligações $\alpha-1,6 .{ }^{19}$

A fava d'anta (Dimorphandra gardneriana Tul e Dimorphandra mollis Benth) é uma planta pertencente à família Legumimosae, e típica do cerrado e da caatinga brasileiras. A espécie Dimorphandra gardneriana é encontrada no estado do Ceará, na Chapada do Ara- 
ripe. ${ }^{20}$ Das sementes pode-se isolar uma galactomanana, com razão manose/galactose de 1,85:1, massa molar média na ordem de $10^{7} \mathrm{~g} /$ mol e com soluções aquosas muito viscosas. ${ }^{21}$ As vagens que contêm as sementes são usadas para produzir rutina, um conhecido flavonoide com propriedades antioxidantes e efeitos anti-inflamatórios. ${ }^{22}$ Para extração deste flavonoide, cerca de 600 t/ano de sementes são descartadas. ${ }^{21}$ As sementes dessas espécies poderiam fornecem cerca de 160 t/ano de galactomanana, ${ }^{21}$ com potencial de inúmeras aplicações em substituição a polissacarídeos importados. ${ }^{23}$

Os objetivos deste trabalho foram estudar as características reológicas de OVDs comerciais brasileiros e, preparar formulações da galactomanana de fava d'anta (Dimorphandra gardeniana Tul) com características reológicas que atendam aos critérios para um eficiente OVD, aliando custo mais baixo e aplicação potencial de recursos naturais da biodiversidade brasileira.

\section{PARTE EXPERIMENTAL}

\section{Materiais}

Três dispositivos viscocirúrgicos oftálmicos (OVD) nacionais foram avaliados, sendo eles: Metilcelulose 2,0\% proveniente da Ophthalmos (São Paulo, SP), Ofthyal ${ }^{\circledR}$ (ácido hialurônico 3\%) da Oft Vision (São Paulo, SP) e Medilon ${ }^{\circledast}$ (poliacrilamida 1,5\%) da Mediphacos Ophthalmic Professionals (Belo Horizonte, MG).

\section{Extração da galactomanana da fava d'anta (Dimorphandra gardneriana $\mathrm{Tul}$ )}

A galactomanana foi extraída a partir de vagens de fava d'anta (Dimorphandra gardneriana Tul) coletadas nos arredores da cidade do Crato (Ceará, Brasil) nos meses de agosto e setembro de 2006. O procedimento de extração foi feito de acordo com a metodologia proposta por Cunha et al. ${ }^{21}$ brevemente descrita a seguir. Uma porção de $20 \mathrm{~g}$ de sementes foi intumescida inicialmente em água fervente e, posteriormente, em água à temperatura ambiente. Os endospermas foram retirados manualmente, intumescidos a quente e triturados. A solução viscosa obtida foi centrifugada e a galactomanana, presente no sobrenadante, precipitada em etanol, lavada com etanol e acetona, e seca.

\section{Preparação das formulações de galactomanana da fava d'anta}

As formulações da galactomanana $(1,0 ; 1,2 ; 1,5 ; 1,8 ; 2,5$ e $3,0 \%$, $\mathrm{m} / \mathrm{v}$ ) foram preparadas em tampão fosfato $0,1 \mathrm{~mol} / \mathrm{L} \mathrm{em} \mathrm{NaCl} 0,15$ $\mathrm{mol} / \mathrm{L}$, sob agitação magnética por $24 \mathrm{~h}$ à temperatura ambiente para completa solubilização.

\section{Estudo reológico dos OVDs comerciais e das formulações de galactomanana}

As medidas reológicas das formulações de fava d'anta e dos OVDs comerciais foram feitas no reômetro AR 550 da TA Instruments, com geometria do tipo cone e placa $(40 \mathrm{~mm})$ à temperatura de $36{ }^{\circ} \mathrm{C}$. A faixa de taxa de cisalhamento aplicada foi de 0,1 a 1000 $\mathrm{s}^{-1}$. As curvas de viscosidade foram ajustadas através de programa TA Advantage Data Analysis, versão 5.0.38, do próprio reômetro. O modelo utilizado foi o de Cross, ${ }^{9}$ que descreve o comportamento reológico de fluidos não-Newtonianos (Equação 1):

$$
\eta=\eta_{\infty}+\frac{\left(\eta_{0}-\eta_{\infty}\right)}{1+(K \dot{\gamma})^{\mathrm{n}}}
$$

onde $\eta_{0}$ e $\eta_{\infty}$ são as viscosidades limites quando a taxa de cisalhamento tende a zero e ao infinito, respectivamente. $\mathrm{K}$ é uma constante de ajuste, $\gamma$ é a taxa de cisalhamento e $n$ é o índice de pseudoplasticidade.

A viscoelasticidade dos OVDs e das formulações foi também avaliada através da determinação dos parâmetros viscoelásticos lineares: G'(módulo de armazenamento em cisalhamento ou módulo elástico) e G" (módulo de perda em cisalhamento ou módulo viscoso). As frequências aplicadas durante o experimento variaram de 0,01 a 10 $\mathrm{Hz}$, mantendo-se a tensão constante em $4 \mathrm{~Pa}$ (faixa de linearidade).

Foram calculados, ainda, o coeficiente de amortecimento ou $\tan \delta$, e a viscosidade dinâmica, $\eta$ '. O primeiro parâmetro está relacionado com a energia dissipada e o segundo, à resistência do gel ao cisalhamento, que é sempre associada com a dissipação interna, geralmente como calor. São calculados pelas Equações 2 e 3: ${ }^{9}$

$$
\tan \delta=\frac{\mathrm{G}^{\prime}}{\mathrm{G}^{\prime}}
$$

$$
\eta^{\prime}=\frac{\mathrm{G}^{\prime}}{\omega}
$$

onde $\omega$ é a velocidade angular.

\section{RESULTADOS E DISCUSSÃO}

\section{Extração da galactomanana da fava d'anta}

O processo de extração apresentou um rendimento de $32,5 \%$ em relação à massa das sementes, comparável ao obtido por Cunha et al... ${ }^{21}$

\section{Reologia de fluxo dos OVDs comerciais e das formulações de galactomanana}

As curvas de fluxo e as de viscosidade para os OVDs nacionais e as formulações de fava d' anta estão mostradas nas Figuras 1 e 2, respectivamente. Observa-se pelas curvas da Figura 1 que a dependência entre a tensão de cisalhamento e a taxa de cisalhamento não é linear em nenhum caso, o que indica um comportamento não-Newtoniano. O tipo de curva mostra um caráter pseudoplástico para todos eles.

Os OVDs testados apresentaram viscosidade na faixa de 2 a 10 Pa.s em taxa de cisalhamento $1 \mathrm{~s}^{-1}$ (Figura 2A). Um OVD muito utilizado internacionalmente, o Viscoat ${ }^{\circledR}$ - produzido pela Alcon Laboratories (Ontário, Canadá), e considerado como "padrão ouro", ${ }^{24}$ apresenta viscosidade de 25 Pa.s, para o mesmo cisalhamento. ${ }^{9}$ Quando se comparam estes valores com os dos OVDs nacionais, verifica-se que eles têm viscosidade menor, o mesmo acontecendo para as formulações de fava d' anta em concentrações abaixo de 1,8\% (Figura 2B).

Comparação mais aprofundada pode ser feita através dos parâmetros calculados pelo ajuste do comportamento das curvas à Equação de Cross (Equação 1). A viscosidade $\eta_{0}$ reflete a habilidade das formulações de manterem a câmara anterior do olho. Quanto maior for $\eta_{0}$ maior será o potencial do material em manter esse espaço., ${ }^{9,25}$ $\mathrm{O}$ índice de pseudoplasticidade, $n$, igual a zero indica um comportamento Newtoniano, indesejável no caso dos OVDs. O valor máximo é 1, comportamento totalmente pseudoplástico. Um valor de $n$ igual a 0,86 foi considerado por Maltese et al. ${ }^{9}$ como um valor adequado para os OVDs.

Analisando-se a Tabela 1 verifica-se que os valores da consistência, K, variam muito entre os OVDs nacionais. O Viscoat ${ }^{\oplus}$ apresenta um valor de $\mathrm{K}$ igual a $0,44,{ }^{9}$ e será utilizado como padrão. As formulações da galactomanana que apresentam valores de $\eta_{0}$, K e $n$ compatíveis, ou seja, $\eta_{0}>57,5$ Pa.s, K próximo de 0,44 e $n$ próximo de 0,86 , são as de concentração 2,5 e 3,0\%. Viscosidades a cisalhamento zero para novos OVDs da classe Healon, ditos visco- 

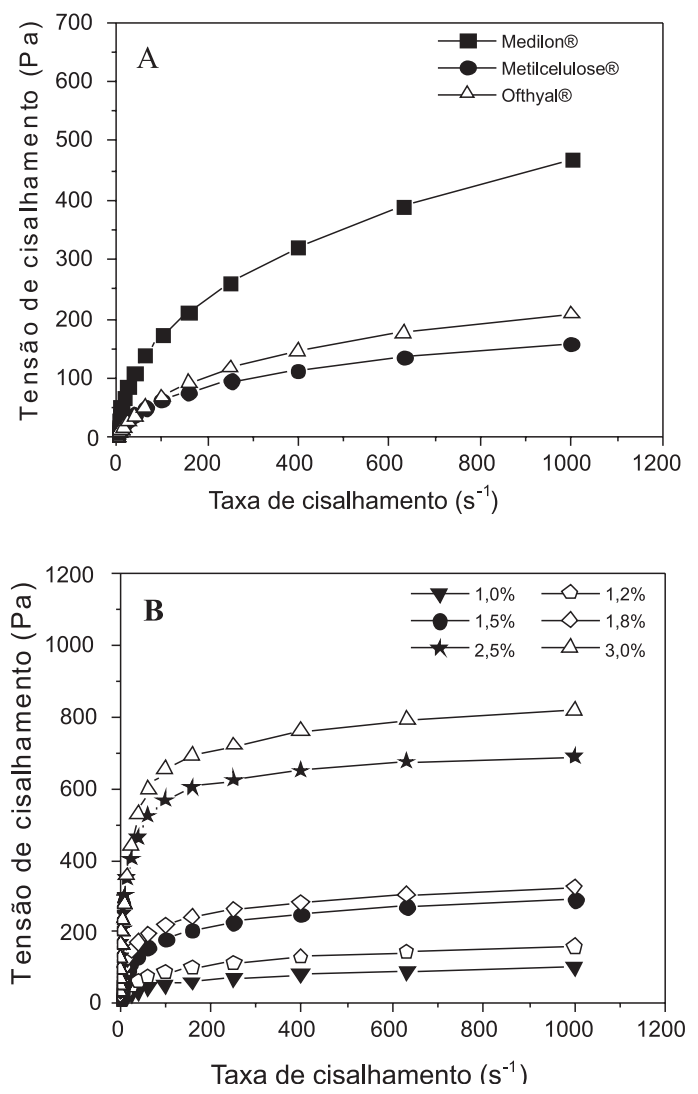

Figura 1. Curva de fluxo a $36{ }^{\circ} \mathrm{C}$ de: a) OVDs comerciais brasileiros; $b$ ) soluções de galactomanana da fava d'anta
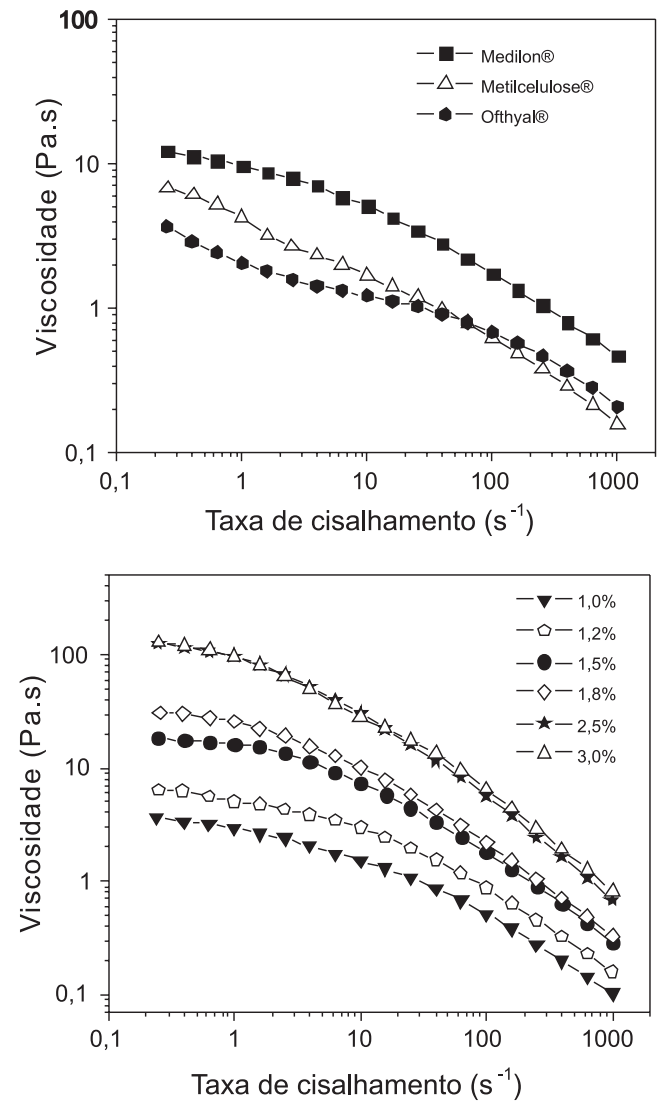

Figura 2. Curva de viscosidade a $36{ }^{\circ} \mathrm{C}$ de: a) OVDs comerciais brasileiros; b) soluções de galactomanana da fava d'anta adaptativos, chegam a 5.525 Pa.s. ${ }^{7}$ Isso indica que $\eta_{0}$ pode ser bem maior do que o apresentado pelo Viscoat e que a solução de fava d'anta $3 \%$ é a mais indicada.

Tabela 1. Parâmetros obtidos pelo modelo de Cross para OVDs comerciais e soluções de galactomanana da fava d'anta

\begin{tabular}{lccc}
\hline Amostra & $\eta_{0}$ (Pa.s) & Consistência, $K$ Índice de pseudoplasticidade, $n$ \\
\hline Medilon $^{\circledR}$ & 14,2 & 0,27 & 0,60 \\
Metilcelulose & 12,9 & 3,98 & 0,51 \\
Ofthyal $^{\circledast}$ & 4,8 & 1,36 & 0,40 \\
Viscoat $^{\circledR 9}$ & 57,5 & 0,44 & 0,86 \\
FD 1,0\% & 4,1 & 0,20 & 0,67 \\
FD 1,2\% & 6,5 & 0,13 & 0,75 \\
FD 1,5\% & 19,6 & 0,18 & 0,81 \\
FD 1,8\% & 34,2 & 0,28 & 0,82 \\
FD 2,5\% & 137,2 & 0,40 & 0,87 \\
FD 3,0\% & 146,9 & 0,49 & 0,82
\end{tabular}

\section{Reologia oscilatória dos OVDs comerciais e formulações da galactomanana}

A variação dos parâmetros viscoelásticos (G' e G”) com o aumento da frequência para os OVDs nacionais e formulações está mostrada nas Figuras 3 e 4. Os valores de G', G' e ๆ' para todas as amostras em determinadas frequências estão exibidos na Tabela 2.

Medilon ${ }^{\circledR}$, Metilcelulose e Ofthyal ${ }^{\circledR}$ apresentam G" maior do que G' em toda a faixa de frequência estudada (Figura 3). Isto significa que eles têm comportamento predominantemente viscoso em toda a faixa de frequência estudada. O comportamento adequado para um OVD é que ele seja viscoso a baixas frequências e elástico a altas. O Viscoat ${ }^{\circledR}$, por exemplo, apresenta-se viscoso até $2 \mathrm{~Hz}$ e elástico de 2 a 10 Hz. .9 Além disso, os valores de G' e de G" são superiores aos respectivos parâmetros dos três OVDs nacionais estudados (Tabela 2).

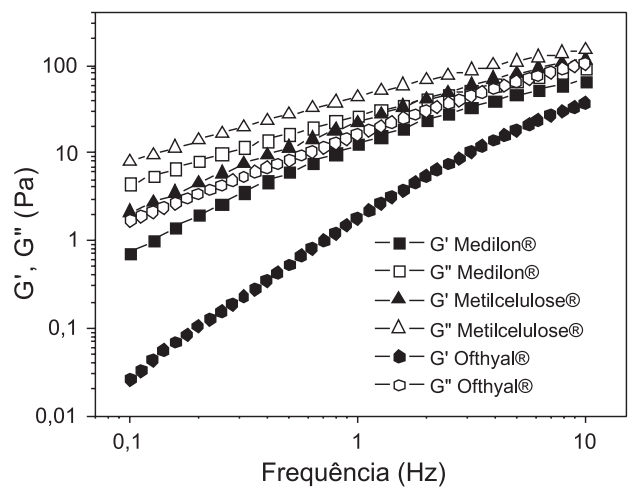

Figura 3. Efeito da frequência oscilatória nos módulos $G^{\prime} e$ " dos OVDs comerciais brasileiros, a $36{ }^{\circ} \mathrm{C}$

As formulações de galactomanana nas concentrações de 1,0 e $1,2 \%$ só apresentam caráter viscoso (Figura 4). Todas as demais apresentam caráter viscoso a frequências mais baixas e caráter elástico a frequências mais altas, adequado a um bom OVD.

Outro parâmetro importante é o ponto de crossover, que é onde G' = G'. . No caso de OVDs, os módulos viscoelásticos no crossover devem ser elevados e a frequência deve ser baixa. ${ }^{7}$ Do ponto de vista clínico, para a implantação adequada da lente intraocular é necessária uma frequência no crossover $\leq 1 \mathrm{rad} / \mathrm{s}$, ou seja $\leq 0,159$ Hz. ${ }^{7} \mathrm{O}$ crossover diminui com o aumento da concentração de fava d'anta, de 2,32 para $0,30 \mathrm{~Hz}$, quando a concentração passa de 1,5 a 3,0\% (Tabela 3). 
Tabela 2. Parâmetros viscoelásticos (G', G' e $\left.\eta^{\prime}\right)$ para OVDs comerciais e soluções de galactomanana da fava d'anta, em frequências representativas

\begin{tabular}{|c|c|c|c|c|}
\hline Amostra & Frequência $(\mathrm{Hz})$ & $\mathrm{G}^{\prime}(\mathrm{Pa})$ & G”(Pa) & $\eta^{\prime}$ (Pa.s) \\
\hline \multirow[t]{4}{*}{ Medilon $^{\circledR}$} & 0,10 & 0,70 & 4,42 & 7,04 \\
\hline & 0,50 & 6,02 & 16,42 & 5,23 \\
\hline & 1,00 & 12,45 & 26,55 & 4,23 \\
\hline & 5,00 & 45,85 & 66,27 & 2,11 \\
\hline \multirow[t]{4}{*}{ Metilcelulose } & 0,10 & 2,08 & 7,82 & 12,45 \\
\hline & 0,50 & 11,51 & 27,33 & 8,70 \\
\hline & 1,00 & 22,27 & 43,62 & 6,94 \\
\hline & 5,00 & 79,93 & 110,00 & 3,50 \\
\hline \multirow[t]{4}{*}{ Ofthyal $^{\circledR}$} & 0,10 & 0,03 & 1,69 & 2,69 \\
\hline & 0,50 & 0,52 & 8,31 & 2,64 \\
\hline & 1,00 & 1,79 & 16,11 & 2,56 \\
\hline & 5,00 & 18,01 & 64,02 & 2,04 \\
\hline \multirow[t]{4}{*}{ Viscoat ${ }^{\circledR 9}$} & 0,10 & 8,46 & 30,23 & 41,00 \\
\hline & 0,50 & 58,14 & 97,61 & 24,00 \\
\hline & 1,00 & 99,49 & 127,00 & 21,00 \\
\hline & 5,00 & 303,00 & 238,00 & 11,00 \\
\hline \multirow[t]{4}{*}{ FD $1,0 \%$} & 0,10 & 0,13 & 1,72 & 2,74 \\
\hline & 0,50 & 2,02 & 7,23 & 2,30 \\
\hline & 1,00 & 4,99 & 12,02 & 1,91 \\
\hline & 5,00 & 20,65 & 27,35 & 0,87 \\
\hline \multirow[t]{4}{*}{ FD $1,2 \%$} & 0,10 & 0,23 & 2,51 & 4,00 \\
\hline & 0,50 & 3,30 & 10,28 & 3,27 \\
\hline & 1,00 & 7,83 & 16,58 & 2,64 \\
\hline & 5,00 & 29,94 & 35,10 & 1,12 \\
\hline \multirow[t]{4}{*}{ FD $1,5 \%$} & 0,10 & 2,95 & 12,18 & 19,39 \\
\hline & 0,50 & 21,41 & 39,69 & 12,63 \\
\hline & 1,00 & 41,15 & 56,98 & 9,07 \\
\hline & 5,00 & 121,30 & 97,55 & 3,10 \\
\hline \multirow[t]{4}{*}{ FD $1,8 \%$} & 0,10 & 5,00 & 20,61 & 32,82 \\
\hline & 0,50 & 37,65 & 64,51 & 20,53 \\
\hline & 1,00 & 70,85 & 89,89 & 14,31 \\
\hline & 5,00 & 190,90 & 138,90 & 4,42 \\
\hline \multirow[t]{4}{*}{ FD $2,5 \%$} & 0,10 & 61,97 & 91,36 & 145,48 \\
\hline & 0,50 & 200,60 & 181,60 & 57,69 \\
\hline & 1,00 & 295,90 & 214,40 & 34,12 \\
\hline & 5,00 & 566,40 & 251,60 & 8,09 \\
\hline \multirow[t]{4}{*}{ FD $3,0 \%$} & 0,10 & 100,00 & 150,20 & 239,17 \\
\hline & 0,50 & 344,00 & 291,70 & 92,67 \\
\hline & 1,00 & 500,40 & 332,70 & 52,95 \\
\hline & 5,00 & 942,50 & 381,50 & 12,27 \\
\hline
\end{tabular}

O tempo de relaxação, $\tau$, reflete a duração do preenchimento efetivo do espaço ocular. Quanto mais tempo durar o preenchimento, melhor. ${ }^{26}$ Em termos clínicos, ele deve ser $\geq 6,3 \mathrm{~s} .{ }^{7}$ Valores da ordem de 80-90 s foram registrados para a linha Healon. ${ }^{7}$ Pela extrapolação da curva de tempo de relaxação versus concentração de fava d'anta, esse valor seria encontrado para uma solução $4,3 \%$.

Elevados valores dos módulos no crossover foram considerados, em termos cirúrgicos, como benéficos..$^{27}$ No entanto, a maioria dos OVDs à base de hialuronato de sódio apresenta módulos de crossover na faixa de 20 a $60 \mathrm{~Pa}^{7}{ }^{7} \mathrm{O}$ padrão Viscoat, com $\mathrm{G}^{\prime}=\mathrm{G}^{\prime \prime}=165 \mathrm{~Pa}$, parece bastante adequado. ${ }^{9}$

Levando em consideração o tempo de relaxação e os módulos viscoelásticos constantes na Tabela 3 verifica-se que a formulação de fava d'anta de 3,0\%, dentre as estudadas, é a que apresenta maior potencial de utilização como OVD.
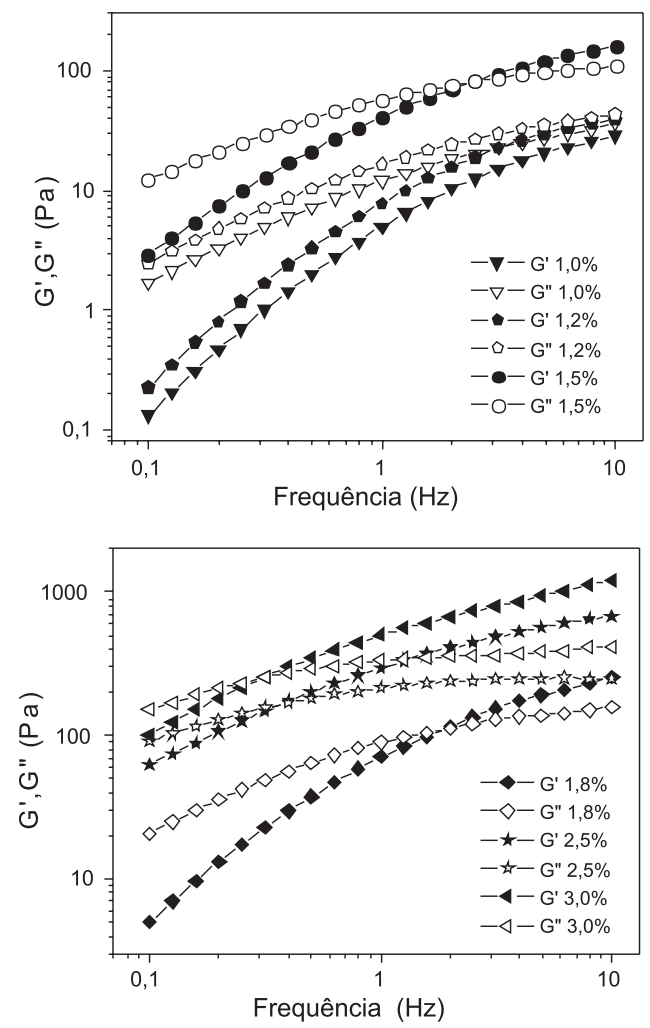

Figura 4. Efeito da frequência oscilatória e da concentração nos módulos $G^{\prime}$ $e$ " das soluções de galactomanana da fava d'anta, a $36{ }^{\circ} \mathrm{C}$

Tabela 3. Frequência e módulos no crossover e tempo de relaxação para os OVDs comerciais e as formulações de fava d'anta

\begin{tabular}{lccc}
\hline OVD & $\begin{array}{c}\mathrm{G}^{\prime}=\mathrm{G}^{\prime} \\
\text { no crossover }(\mathrm{Pa})\end{array}$ & $\begin{array}{c}\text { Frequência de } \\
\text { crossover }(\mathrm{Hz})\end{array}$ & $\begin{array}{c}\text { Tempo de } \\
\text { relaxação (s) }\end{array}$ \\
\hline Medilon $^{\circledR}$ & - & - & - \\
Metilcelulose $^{\text {Ofthyal }}$ & - & - & - \\
Viscoat $^{\circledR 9}$ & - & - & - \\
FD 1,0\% & 165 & 2,0 & 0,5 \\
FD 1,2\% & - & - & - \\
FD 1,5\% & - & - & - \\
FD 1,8\% & 78 & 2,32 & 0,43 \\
FD 2,5\% & 111 & 1,88 & 0,53 \\
FD 3,0\% & 162 & 0,37 & 2,70 \\
\hline
\end{tabular}

A dependência dos módulos viscoelásticos no crossover para soluções aquosas de hialuronato de sódio em tampão fosfato/salina a $23^{\circ} \mathrm{C}$ foi determinada como $\left(\mathrm{G}^{\prime}=\mathrm{G}^{\prime \prime}\right)_{\text {crossover }} \alpha \mathrm{c}^{2,1 \pm 0,1} \mathrm{M}^{0}$. ${ }^{7}$ No caso das soluções de fava d' anta a dependência com a concentração foi de $\left(G^{\prime}=G^{\prime}\right)_{\text {crossover }} \alpha \mathrm{c}^{1,6}$, com boa correlação linear da curva de $\log \mathrm{G}$ versus $\log \mathrm{c}(\mathrm{R}=0,991)$.

\section{Comparação final}

Um eficiente OVD deve apresentar as seguintes características reológicas: viscosidade em cisalhamento zero $\geq 58 \mathrm{~Pa}$.s (se possível bem maior); índice de pseudoplasticidade $\geq 0,86$; módulos viscoelásticos no crossover $\geq 165 \mathrm{~Pa}$; módulo elástico em $0,1 \mathrm{~Hz} \geq 8 \mathrm{~Pa}$; tempo de relaxação $\geq 6,3 \mathrm{~s}$. A Tabela 4 apresenta uma visão de todos os parâmetros reológicos importantes para os OVDs. Nenhum dos OVDs brasileiros estudados pode ser considerado como eficiente. Das formulações com a galactomanana da fava d'anta estudadas, a de concentração 3,0\% é a que apresenta maior potencial de uso como viscoelástico oftálmico. 
Tabela 4. Comparação geral entre os OVDs comerciais e as formulações de fava d'anta

\begin{tabular}{lcccccc}
\hline Amostra & $\begin{array}{c}\eta_{0} \\
(\mathrm{~Pa} . \mathrm{s})\end{array}$ & $\mathrm{n}$ & $\begin{array}{c}\mathrm{G}^{\prime}(\mathrm{Pa}) \\
\mathrm{em} 0,1 \mathrm{~Hz}\end{array}$ & $\begin{array}{c}\mathrm{G}^{\prime \prime}(\mathrm{Pa}) \\
\text { em 0,1 Hz }\end{array}$ & $\begin{array}{c}\mathrm{G}^{\prime}=\mathrm{G}^{\prime \prime} \\
\text { crossover }(\mathrm{Pa})\end{array}$ & $\tau(\mathrm{s})$ \\
\hline Medilon $^{\circledR}$ & 14,2 & 0,60 & 0,70 & 4,42 & - & - \\
Metilcelulose $^{12,9}$ & 0,51 & 2,08 & 7,82 & - & - \\
Ofthyal $^{\circledR}$ & 4,8 & 0,40 & 0,03 & 1,69 & - & - \\
Viscoat $^{\circledR 9}$ & 57,5 & 0,86 & 8,45 & 30,23 & 165 & 0,50 \\
Healon $^{\circledR 7,28}$ & 243 & nd & 26 & nd & 19,4 & 21,4 \\
FD 1,0\% & 4,1 & 0,67 & 0,13 & 1,72 & - & - \\
FD 1,2\% & 6,5 & 0,75 & 0,23 & 2,51 & - & - \\
FD 1,5\% & 19,6 & 0,81 & 2,95 & 12,18 & 78 & 0,43 \\
FD 1,8\% & 34,2 & 0,82 & 5,00 & 20,61 & 111 & 0,53 \\
FD 2,5\% & 137,2 & 0,87 & 61,97 & 91,36 & 162 & 2,70 \\
FD 3,0\% & 146,9 & 0,82 & 100,0 & 150,2 & 250 & 3,33 \\
\hline
\end{tabular}

nd = não disponível

Outros parâmetros, além dos viscoelásticos, são importantes, principalmente os testes in vivo. Eles serão objeto de um estudo posterior.

\section{CONCLUSÃO}

As seguintes conclusões são oriundas do estudo reológico: os dispositivos viscoelásticos oftálmicos produzidos no Brasil (Medilon ${ }^{\circledR}$, Metilcelulose e Ofthyal ${ }^{\circledR}$ ) apresentam características reológicas muito inferiores às requeridas para um eficiente OVD; a formulação de galactomanana da fava d'anta na concentração de $3,0 \%$ é a que apresenta potencial de utilização como dispositivo viscocirúrgico oftálmico.

\section{MATERIAL SUPLEMENTAR}

O gráfico de tan $\delta$ versus frequência, que indica claramente onde ocorre o crossover (uma vez que é onde tan $\delta$ é igual à unidade), está disponível como Figura 1S, em http://quimicanova.sbq.org.br, na forma de arquivo .PDF, com acesso livre.

\section{AGRADECIMENTOS}

Ao CNPq (Rede Nanoglicobiotec - CNPq) e ao INOMAT: INCT de Materiais Complexos Funcionais pelo suporte financeiro e à FUNCAP pela Bolsa de Iniciação Científica.

\section{REFERÊNCIAS}

1. World Health Organization; Prevention of blindness and visual impairment: cataract, disponível em http://www.who.int/blindness/ causes/priority/en/index1.html, acessada em Julho 2009 e Agosto 2010.
2. Oliveira, D. F.; Lira, R. P. C.; Lupinacci, A. P. C.; Paccola, M.; Arieta, C. E. L.; Cad. Saúde Pública 2008, 24, 2440.

3. Gomes, B. A. F.; Biancardi, A. L.; Netto, C. F.; Gaffree, F. F. P.; Moraes Junior, H. V.; Rev. Bras. Oftalmol. 2008, 67, 220.

4. Santhiago, M. R.; Gomes, B. A. F.; Gaffree, F. F. P.; Varandas, V. S.; Costa Filho, A. A.; Rev. Bras. Oftalmol. 2009, 68, 13.

5. Olson, R. J.; Mamalis, N.; Werner, L.; Apple, D. J.; Am. J. Ophthalmol. 2003, 136, 146.

6. Maar, N.; Graebe, A.; Schild, G.; Stur, M.; Amon, M.; J. Cataract Refract. Surg. 2001, 27, 1756.

7. Calciu-Rusu, D.; Rothfuss, E.; Eckelt, J.; Haase, T.; Dick, H. B.; Wolf, B.; Biomacromolecules 2007, 8, 1287.

8. Arshinoff, S. A.; Wong, E.; J. Cataract Refract. Surg. 2003, 29, 2318.

9. Maltese, A.; Bozacchiello, A.; Mayol, L.; Bucolo, C.; Maugeri, F.; Nicolais, L.; Ambrosio, L.; Biomaterials 2006, 27, 5134.

10. Arshinoff, S. A.; J. Cataract Refract. Surg. 1999, 25, 167.

11. http://www.internationalpharmacy.com/pt/products/details, acessada em Agosto de 2010.

12. http://www.medshopexpress.com/10048476.html, acessada em Outubro 2009 e Outubro 2010.

13. http://compare.ebay.com/like/370366232552, acessada em Agosto de 2010.

14. Louzada, N.; Fontes, P. C.; Informativo da Cooeso 2003.

15. Stumpf, S.; Nosé, W.; Arq. Bras. Oftalmol. 2006, 69, 491.

16. Pereira, A. E.; Pereira, A. C. A.; Ávila, M. P.; Arq. Bras. Oftalmol. 2008, $71,695$.

17. Informação do representante da Ophthalmos na cidade de São Paulo em 9 de agosto de 2010.

18. Informação do setor de vendas da Mediphacos Ophtalmic Professional em Belo Horizonte, MG, em 09 de agosto de 2010.

19. Stephen, A. M.; Food polysaccharides and their applications, Marcel Dekker: Nova York, 1995.

20. http://florabrasiliensis.cria.org.br, acessada em Março 2008 e Agosto 2010.

21. Cunha, P. L. R.; Vieira, I. G. P.; Arriaga, A. M. C.; de Paula, R. C. M.; Feitosa, J. P. A.; Food Hydrocolloids 2008, 23, 880.

22. http://www.phytochemicals.info/phytochemicals/Rutin, acessada em Março 2008 e Agosto 2010.

23. Cunha, P. L. R.; de Paula, R. C. M.; Feitosa, J. P. A.; Quim. Nova 2009, $32,649$.

24. Peck, C. M. C.; Joos, Z. P.; Zaugg, B. E.; Abdel-Aziz, S.; Stringham, J. D.; Werner, L.; Mamalis, N.; Olson, R. J.; Clin. Exp. Ophthalmol. 2009, 37, 397.

25. Andrews, G. P.; Gorman, S. P.; Jones, D. S.; Biomaterials 2005, 26, 571.

26. Dick, H. B.; Krummenauer, F.; Augustin, A. J.; Pakula T.; Pfeiffer, N.; J. Cataract Refract. Surg. 1999, 25, 1630.

27. Floyd, M.; Valentine, J.; Coombs, J.; Olson, R. J.; J. Cataract Refract. Surg. 2006, 32, 1222.

28. Dick, H. B.; Krummenauer, F.; Augustin, A. J.; Pakula T.; Pfeiffer, N.; J. Cataract Refract. Surg. 2001, 27, 320. 


\section{VISCOELÁSTICOS OFTÁLMICOS: COMPARAÇÃO ENTRE OS COMERCIAIS E FORMULAÇÕES DE}

GALACTOMANANA DE Dimorphandra gardneriana

Natália R. Pires, Pablyana L. R. da Cunha, Regina C. M. de Paula e Judith P. A. Feitosa*

Departamento de Química Orgânica e Inorgânica, Universidade Federal do Ceará, CP 6021, 60455-760, Fortaleza - CE, Brasil Francisco V. F. Jamacaru e Manoel O. Moraes Filho

Depto de Fisiologia e Farmacologia, Universidade Federal do Ceará, 60430-270 Fortaleza - CE, Brasil

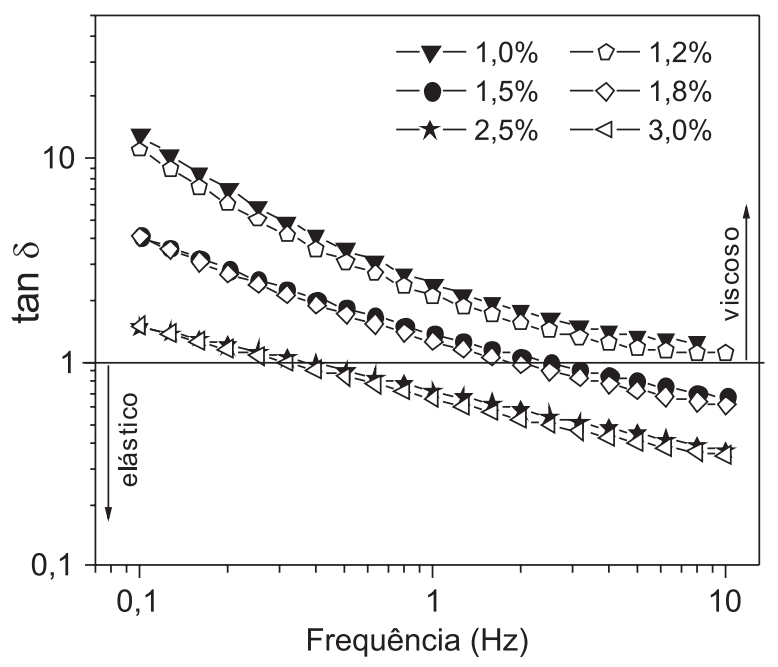

Figura 1S. Efeito da frequência oscilatória e da concentração na tangente de perda das soluções de galactomanana da fava d'anta, a $36^{\circ} \mathrm{C}$ 